\title{
Some Notes on the Paper "New Common Fixed Point Theorems for Maps on Cone Metric Spaces”
}

\author{
Mohamed Abd El-Rahman Ahmed \\ Department of Mathematics, Faculty of Science, Assiut University, Assiut, Egypt \\ Email: mahmed68@yahoo.com
}

Received January 17, 2013; revised February 21, 2013; accepted March 19, 2013

Copyright (C) 2013 Mohamed Abd El-Rahman Ahmed. This is an open access article distributed under the Creative Commons Attribution License, which permits unrestricted use, distribution, and reproduction in any medium, provided the original work is properly cited.

\begin{abstract}
In this paper, we show that Theorem 2.1 [1] (resp. Theorem 2.2 [1]) is a consequence of Corollary 2.1 [1] ( resp. Corollary $2.2[1])$.
\end{abstract}

Keywords: Cone Metric; Weakly Compatible; Fixed Point

\section{Introduction}

In 2007, Huang and Zhang [2] initiated fixed point theory in cone metric spaces. On the other hand, in 2011, Haghi, Rezapour and Shahzad [3] gave a lemma and showed that some fixed point generalizations are not real generalizations. In this note, we show that Theorem 2.1 [1] and Theorem 2.2 [1] are so.

Following [2], let $E$ be a real Banach space and $\theta$ be the zero vector in $E$, and $P \subseteq E . P$ is called cone iff

1) $P$ is closed, nonempty and $P \neq\{\theta\}$,

2) $a x+b y \in P$ for all $x, y \in P$ and nonnegative real numbers $a, b$,

3) $P \cap(-P)=\{\theta\}$.

For a given cone $P$, we define a partial ordering $\preceq$ with respect to $P$ by $x \preceq y$ iff $y-x \in P . x \prec y$ (resp. $x \square y$ ) stands for $x \preceq y$ and $x \neq y$ (resp. $y-x$ $\in \operatorname{int}(P)$ ), where $\operatorname{int}(P)$ denotes the interior of $P$. In the paper we always assume that $P$ is solid, i.e., $\operatorname{int}(P) \neq \phi$. It is clear that $x \square y$ leads to $x \preceq y$ but the reverse need not to be true.

The cone $P$ is called normal if there exists a number $K>0$ such that for all $x, y \in E, \theta \preceq x \preceq y$ implies $\|x\| \leq K\|y\|$.

The least positive number satisfying above is called the normal constant of $P$.

Definition 1.1 [2]. Let $X$ be a nonempty set. A function $d: X \times X \rightarrow E$ is called cone metric iff

$$
\begin{aligned}
& \left(\mathrm{M}_{1}\right) \quad \theta \preceq d(x, y), \\
& \left(\mathrm{M}_{2}\right) d(x, y)=d(y, x)=\theta \text { iff } x=y,
\end{aligned}
$$

$$
\begin{aligned}
& \left(\mathrm{M}_{3}\right) d(x, y)=d(y, x), \\
& \left(\mathrm{M}_{4}\right) d(x, y) \preceq d(x, z)+d(z, y),
\end{aligned}
$$

for all $x, y, z \in X .(X, d)$ is said to be a cone metric space.

Lemma 1.1 [3]. Let $X$ be a nonempty and $f: X \rightarrow X$. Then there exists a subset $Y \subseteq X$ such that $f(Y)=f(X)$ and $f: Y \rightarrow X$ is one-to-one.

Definition 1.2 [4]. Let $(X, d)$ be a cone metric space and $f, g: X \rightarrow X$ be mappings. Then, $z \in X$ is called a coincidence point of $f$ and $g$ iff $f(z)=g(z)$.

Definition 1.3 [4]. Let $(X, d)$ be a cone metric space. The mappings $f, g: X \rightarrow X$ are weakly compatible iff for every coincidence point $z \in X$ of $f$ and $g$, $f(g(x))=g(g(x))$.

Theorem 1.1 (Theorem 2.1 [1]). Let $(X, d)$ be a cone metric space and let $a_{i} \geq 0 \quad(i=1,2,3,4,5)$ be constants with $a_{1}+a_{2}+a_{3}+a_{4}+a_{5}<1$. Suppose that the mappings $f, g: X \rightarrow X$ satisfy the condition

$$
\begin{aligned}
& d(f(x), f(y)) \preceq a_{1} d(g(x), g(y)) \\
& +a_{2} d(f(x), g(x))+a_{3} d(f(y), g(y)) \\
& +a_{4} d(g(x), f(y))+a_{5} d(f(x), g(y))
\end{aligned}
$$

for all $x, y \in X$.

If the range of $g$ contains the range of $f$ and $g(X)$ is a complete subspace, then $f$ and $g$ have a unique point of coincidence in $X$. Moreover, if $f$ and $g$ are weakly compatible, then $f$ and $g$ have a unique fixed point.

Theorem 1.2 (Corollary 2.1 [1]). Let $(X, d)$ be a complete cone metric space and let $a_{i} \geq 0 i=(1,2,3,4,5)$ 
be constants with $a_{1}+a_{2}+a_{3}+a_{4}+a_{5}<1$. Suppose that the mapping $f: X \rightarrow X$ satisfies the condition

$$
\begin{aligned}
& d(f(x), f(y)) \preceq a_{1} d(x, y)+a_{2} d(x, f(x)) \\
& +a_{3} d(y, f(y))+a_{4} d(x, f(y))+a_{5} d(y, f(x))
\end{aligned}
$$

for all $x, y \in X$.

Then $f$ has a unique fixed point $x^{*}$ in $X$.

Theorem 1.3 (Theorem 2.2 [1]). Let $(X, d)$ be a cone metric space and let the mappings $f, g: X \rightarrow X$ satisfy the condition

$$
d(f(x), f(y)) \preceq \lambda \cdot u, \text { for all } x, y \in X,
$$

where

$$
\begin{aligned}
& u \in\{d(g(x), g(y)), d(f(x), g(x)), d(f(y), g(y)), \\
& \left.\frac{1}{h}[d(f(x), g(y))+d(f(y), g(x))]\right\},
\end{aligned}
$$

$\lambda \in(0,1), h>2 \lambda$.

If the range of $g$ contains the range of $f$ and $g(X)$ is a complete subspace, then $f$ and $g$ have a unique point of coincidence in $X$. Moreover, if $f$ and $g$ are weakly compatible, then $f$ and $g$ have a unique fixed point.

Theorem 1.4 (Corollary 2.2 [1]). Let $(X, d)$ be a complete cone metric space and let the mapping $f: X \rightarrow X$ satisfies the condition

$$
d(f(x), f(y)) \preceq \lambda \cdot u, \text { for all } x, y \in X,
$$

where

$$
\begin{gathered}
u \in\{d(x, y), d(f(x), x), d(f(y), y), \\
\left.\frac{1}{h}[d(f(x), y)+d(f(y), x)]\right\},
\end{gathered}
$$

$\lambda \in(0,1), h>2 \lambda$.

Then $f$ has a unique fixed point $x^{*}$ in $X$.

\section{Main Result}

In this section, we show that that Theorem 1.1 (resp. Theorem 1.3) is a consequence of Theorem 1.2 (resp. Theorem 1.4).
Theorem 2.1. Theorem 1.1 is a consequence of Theorem 1.2.

Proof. By Lemma 1.1, there exists $Y \subseteq X$ such that $g(Y)=g(X)$ and $g: Y \rightarrow X$ is one-to-one. Define a map $h: g(Y) \rightarrow g(Y)$ by $h(g(x))=f(x)$ for each $x \in g(Y)$. Since $g$ is one-to-one on $Y$, then $h$ is well-defined. Also, for arbitrary $x, y \in X$,

$$
\begin{aligned}
& d(h(g(x)), h(g(y))) \preceq a_{1} d(g(x), g(y)) \\
& +a_{2} d(h(g(x)), g(x))+a_{3} d(h(g(y)), g(y)) \\
& +a_{4} d(g(x), h(g(y)))+a_{5} d(h(g(x)), g(y))
\end{aligned}
$$

where $a_{i} \geq 0 \quad(i=1,2,3,4,5)$ are constants with

$$
a_{1}+a_{2}+a_{3}+a_{4}+a_{5}<1 .
$$

From the completeness of $g(Y)=g(X)$, there exists $x_{0} \in X$ such that

$$
h\left(g\left(x_{0}\right)\right)=g\left(x_{0}\right)=f\left(x_{0}\right)
$$

by Theorem 1.2. Hence, $f$ and $g$ have a point of coincidence which is also unique. Since $f$ and $g$ are weakly compatible, then $f$ and $g$ have a unique common fixed point.

Theorem 2.2. Theorem 1.3 is a consequence of Theorem 1.4.

\section{REFERENCES}

[1] G. Song, X. Sun, Y. Zhao and G. Wang, "New Common Fixed Point Theorems for Maps on Cone Metric Spaces," Applied Mathematics Letters, Vol. 23, No. 9, 2010, pp. 1033-1037. doi:10.1016/j.aml.2010.04.032

[2] L.-G. Huang and X. Zhang, "Cone Metric Spaces and Fixed Point Theorems of Contractive Mappings,” Journal of Mathematical Analysis and Applications, Vol. 332, No. 2, 2007, pp. 1468-1476. doi:10.1016/j.jmaa.2005.03.087

[3] R. H. Haghi, Sh. Rezapour and N. Shahzad, "Some Fixed Point Generalizations Are Not Real Generalizations," Nonlinear Analysis, Theory, Methods and Applications, Vol. 74, 2011, pp. 1799-1803.

[4] C. Di Bari and P. Vetro, “ $\phi$-Pairs and Common Fixed Points in Cone Metric Spaces,” Rendiconti del Circolo Matematico di Palermo, Vol. 57, No. 2, 2008, pp. 279285. doi:10.1007/s12215-008-0020-9 